\title{
LA COMPÉTITION INTERSPÉCIFIQUE ENTRE LA TRUITE COMMUNE (SALMO TRUTTA L.) ET LA TRUITE ARC-EN-CIEL (ONCORHYNCHUS MYKISS WALBAUM) : INFLUENCE SUR LES MODĖLES D'HABITAT.
}

\author{
P. BARAN, M. DELACOSTE, J.M. LASCAUX, F. DAUBA, G. SEGURA
}

Laboratoire d'Ingéniérie Agronomique, Equipe Environnement Aquatique et Aquaculture,

Ecole Nationale Supérieure Agronomique de Toulouse, 145 av. de Muret, 31076 Toulouse Cedex, France.

\section{RÉSUMÉ}

L'influence de la compétition interspécifique sur l'occupation de l'habitat physique du ruisseau d'Estibère a été étudiée sur deux populations de truite arc-en-ciel (Oncorhynchus mykiss), vivant respectivement en allopatrie et en sympatrie avec une population de truite commune (Salmo trutta L.). Le taux d'occupation de la Surface Pondérée Utile (SPU) calculée par la méthode des microhabitats (BOVEE, 1982) est significativement supérieur (t de Student, $p<0.05$ ) pour la population allopatrique de truite arc-en-ciel par rapport à la population sympatrique. La surface d'abris, la pente et le taux d'occupation de la SPU par les truites communes permettent d'expliquer $77 \%$ de la variation d'occupation de l'habitat physique par les adultes de truites arc-en-ciel. Pour les truites communes, la surface d'abris explique à elle seule, $81 \%$ de la variation du taux d'occupation de l'habitat par les adultes. Selon l'espèce et le stade de développement considéré, la compétition interspécifique peut être un élément déterminant dans l'occupation de l'habitat physique potentiel.

Mots-clés : truite commune, truite arc-en-ciel, Salmo trutta, Oncorhynchus mykiss, compétition, habitat.

\section{THE INTERSPECIFIC COMPETITION BETWEEN BROWN TROUT (SALMO TRUTTA L.) AND RAINBOW TROUT (ONCORHYNCHUS MYKISS WALBAUM) : INFLUENCE ON HABITAT MODELS.}

\begin{abstract}
The influence of interspecific competition on the occupation of physical habitat of the Estibere stream was studied in two rainbow trout populations (Oncorhynchus mykiss), one in allopatry and the other in sympatry with brown trout (Salmo trutta L.). The occupation of the Weighted Usable Area (WUA) was calculated by the PHABSIM model of the IFIM methodology (BOVEE, 1982). The occupation by rainbow trouts was significantly greater (t test, $p<0.05$ ) for the allopatric population than for the sympatric population. Surface of cover, gradient and the occupation of the physical habitat by adults of brown trout explained $77 \%$ of the variation of the occupation of the physical habitat by rainbow trout. For the adults of brown trout, the surface of cover explained $81 \%$ of the variation of the occupation of physical habitat. Interspecific competition may influence the occupation of physical habitat by the different species and life-stages.
\end{abstract}

Key-words : brown trout, rainbow trout, Salmo trutta, Oncorhynchus mykiss, competition, habitat.

\section{INTRODUCTION}

La compétition inter et intraspécifique est un élément déterminant dans la stabilité et la complexité des communautés biologiques. Au niveau des Salmonidés, les nombreuses introductions ont créé des combinaisons d'espèces très variées. FAUSCH (1988) estime à 
78 le nombre de combinaisons potentielles sur le continent nord-américain. L'introduction de nouvelles espèces peut fortement perturber une espèce indigène. Ainsi, la truite commune a fortement supplanté l'omble de fontaine dans de nombreuses rivières des Etats-Unis (WATERS, 1983 ; FAUSCH et WHITE, 1986). Les processus par lesquels s'exprime la compétition sont relativement complexes et se traduisent souvent par des changements ou des réductions de niches écologiques (GATZ et al., 1987). Les caractéristiques d'habitat peuvent interférer avec ces processus et notamment la pente de la rivière (FAUSCH, 1989 ; BOZEK et HUBERT, 1992). Le vallon d'Estibère constitue un milieu montagnard d'une richesse écologique remarquable. Du point de vue piscicole, il présente la particularité d'accueillir deux espèces de truite : la truite commune (Salmo trutta L.) et la truite arc-en-ciel (Oncorhynchus mykiss Walbaum). II existe des données de référence sur ce secteur (ELLIOTT, 1973 ; DUMAS, 1976). L'objectif de l'étude est d'analyser l'effet de la compétition interspécifique sur l'occupation de l'habitat physique du chenal et les interférences avec les autres caractéristiques de l'habitat.

\section{PRÉSENTATION DU SITE ET MÉTHODOLOGIE}

Le ruisseau d'Estibère est situé en totalité dans la réserve naturelle du Néouvielle (réserve de pêche intégrale). II appartient au bassin de la Neste d'Aure, principal affluent pyrénéen de la Garonne. Ce ruisseau, d'une longueur de $3.5 \mathrm{kms}$ et d'une largeur moyenne de $3 \mathrm{~m}$, alterne des tronçons de forte pente avec des tronçons de plateau (tourbière). Les eaux sont faiblement minéralisées (conductivité : $40 \mu \mathrm{S} / \mathrm{cm}$ ), la température estivale y est élevée $\left(13.5{ }^{\circ} \mathrm{C}\right.$ en moyenne) tandis que la température hivernale est très froide $\left(<1^{\circ} \mathrm{C}\right)$. Du point de vue piscicole, on distinque deux zones : l'une en amont avec une population allopatrique de truite arc-en-ciel (Oncorhynchus mykiss) introduite en 1958 dans le vallon, et l'autre en aval avec deux populations sympatriques de truite arc-en-ciel et de truite commune (Salmo trutta L.). Ces deux zones sont isolées par une rupture de pente $(>30 \%$ ) infranchissable par les poissons de l'aval. Depuis 1958, la truite arc-en-ciel se maintient sans aucun déversement.

Dix-sept stations d'étude ont été prospectées en juillet 1992 et 1993. Les populations piscicoles, l'habitat physique (vitesse, profondeur, substrat et abris) et la qualité physicochimique de l'eau (température, conductivité, nitrates) ont été échantillonnés en période de débit d'étiage estival, selon les protocoles utilisés par BARAN et al. (1993a et 1993b). Pour chaque station et pour chaque espèce la Surface Pondérée Utile (SPU) a été calculée par la méthode des microhabitats après un découpage par cellule à partir des mesures de vitesses, de profondeurs et de granulométrie (BOVEE, 1982 ; BELAUD et al., 1989), selon la formule :

$$
S P U=\sum_{i=1}^{n} P\left(V_{i}\right) \times P\left(P r_{i}\right) \times A_{i}
$$

avec $P\left(V_{i}\right)=$ Valeur de préférence pour la vitesse de la cellule $i$.

$P\left(P f_{j}\right)=$ Valeur de préférence pour la profondeur de la cellule ${ }_{j}$.

$P\left(S_{i}\right)=$ Valeur de préférence pour la granulométrie du substrat de la cellule $i$.

$A_{i} \quad=$ Surface de la cellule $i$.

Pour la truite commune, les courbes de préférences établies par BELAUD et al. (1989) sur les rivières du sud-ouest de la France ont été utilisées tandis que pour la truite arc-en-ciel, ce sont celles proposées par LOAR et al. (1985) qui ont été utilisées. Des taux d'occupation $(T)$ de l'habitat physique potentiel déterminé par la méthode des microhabitats ont ensuite été calculés selon la formule :

$$
T=\text { Abondance / SPU }
$$

où l'abondance est en $\mathrm{g}$ ou en nombre d'individidus et la SPU en $\mathrm{m}^{2}$.

En référence aux courbes de préférences, les poissons de taille inférieure à $100 \mathrm{~mm}$ ont été considérés comme alevins, entre 100 et $159 \mathrm{~mm}$ comme juvéniles, et supérieure ou égale à $160 \mathrm{~mm}$ comme adultes. 
La variabilité de ces taux d'occupation a été analysée à partir d'une procédure de régression multiple progressive (WEISBERG,1980). Différentes variables susceptibles d'influencer cette variabilité ont été introduites dans la procédure (la pente, les surfaces d'abris, le taux d'occupation par la seconde espèce de Salmonidés présente ou par un autre stade de développement de la même espèce).

\section{RÉSULTATS}

\section{Abondances des deux espèces}

Les densités de truites arc-en-ciel dans les différents échantillonnages réalisés sur le vallon d'Estibère (ELLIOT, 1973 ; DUMAS, 1976) ont fortement régressé. Cette espèce représentait $99,5 \%$ de la densité totale en $1968,74 \%$ en 1972 et seulement $26 \%$ en 1992 sur une même station.

En situation de sympatrie, les biomasses et densités de truite arc-en-ciel sont significativement inférieures à celles de la truite commune ( $t$ de student, $p<0.05$ ) (tableaux I et II). En moyenne, les truites arc-en-ciel représentent 35,8\% de la biomasse totale et $31,6 \%$ de la densité. Les densités relatives sont significativement corrélées à la pente de la station de truites arc-en-ciel $(r=0.72, p<0.01)$.

Tableau I : Caractéristiques d'abondance des populations de truite arc-en-ciel dans le ruisseau d'Estibère (densité : ind./ha, biomasse : $\mathrm{kg} / \mathrm{ha}$ ).

Table I : Abundance characteristics of rainbow trout populations in the Estibere stream (density : ind./ha, biomass : $\mathrm{kg} / \mathrm{ha}$ ).

Truite arc-en-ciel

Stations Alevin (<100 mm) Juvénile $(100-160 \mathrm{~mm}) \quad$ Adulte $(>160 \mathrm{~mm})$ Densité Biomasse Densité Biomasse Densité Biomasse

\begin{tabular}{rrrrrrr}
\hline$I$ & 0 & 0.0 & 384 & 10 & 578 & 40 \\
$I I$ & 0 & 0.0 & 139 & 4 & 278 & 20 \\
III & 0 & 0.0 & 0 & 0 & 0 & 0 \\
IV & 0 & 0.0 & 264 & 9 & 264 & 17 \\
$V$ & 159 & 0.9 & 159 & 5 & 159 & 11 \\
VI & 147 & 0.7 & 294 & 10 & 441 & 33 \\
VII & 0 & 0.0 & 636 & 23 & 1059 & 62 \\
VIII & 0 & 0.0 & 0 & 0 & 1015 & 80 \\
IX & 0 & 0.0 & 0 & 0 & 256 & 12 \\
$X$ & 245 & 1.6 & 900 & 27 & 409 & 22 \\
XI & 521 & 0.5 & 260 & 10 & 521 & 31 \\
XII & 659 & 4.9 & 329 & 11 & 1207 & 81 \\
XIII & 585 & 9.3 & 987 & 30 & 914 & 65 \\
XIV & 251 & 1.7 & 1380 & 43 & 1254 & 81 \\
XV & 782 & 6.0 & 1565 & 47 & 1330 & 95 \\
XVI & 473 & 3.7 & 947 & 27 & 861 & 67 \\
XVII & 750 & 5.6 & 416 & 10 & 750 & 69 \\
\hline
\end{tabular}


Tableau If : Caractéristiques d'abondance des populations de truite commune dans le ruisseau d'Estibère (densité : ind./ha, biomasse : $\mathrm{kg} / \mathrm{ha}$ ).

Table II : Abundance characteristics of brown trout populations in the Estibere stream (density : ind./ha, biomass : $\mathrm{kg} / \mathrm{ha}$ ).

\begin{tabular}{|c|c|c|c|c|c|c|}
\hline \multirow[t]{2}{*}{ Stations } & \multicolumn{2}{|c|}{ Alevin (<100 mm) } & \multicolumn{2}{|c|}{$\begin{array}{c}\text { Truite commune } \\
\text { Juvénile }(100-160 \mathrm{~mm})\end{array}$} & \multicolumn{2}{|c|}{ Adulte $(>160 \mathrm{~mm})$} \\
\hline & Densité & Biomasse & Densité & Biomasse & Densité & Biomasse \\
\hline I & 0 & 0.0 & 1623 & 41 & 865 & 46 \\
\hline$\|$ & 117 & 0.7 & 352 & 12 & 235 & 14 \\
\hline III & 105 & 1.2 & 421 & 14 & 211 & 9 \\
\hline IV & 0 & 0.0 & 768 & 16 & 1811 & 95 \\
\hline V & 1078 & 6.8 & 2772 & 89 & 689 & 34 \\
\hline VI & 232 & 0.8 & 2098 & 57 & 697 & 32 \\
\hline VII & 0 & 0.0 & 192 & 5 & 0 & 0 \\
\hline VIII & 252 & 1.2 & 2014 & 52 & 1007 & 48 \\
\hline IX & 769 & 5.1 & 1026 & 28 & 0 & 0 \\
\hline$x$ & 0 & 0.0 & 1591 & 36 & 924 & 42 \\
\hline$X \mid$ & 294 & 3.3 & 294 & 10 & 586 & 30 \\
\hline
\end{tabular}

En comparant les deux situations amont et aval du ruisseau, on remarque que les densités et biomasses de truite arc-en-ciel sont significativement inférieures en situation de sympatrie (t de student, $\mathrm{p}<0.05$ ) par rapport à une situation d'allopatrie.

\section{Taux d'occupation de I'habitat physique}

Pour les truites arc-en-ciel, les taux d'occupation de l'habitat physique sont significativement inférieurs en situation de sympatrie par rapport à une situation d'allopatrie, quel que soit le stade de développement considéré ( $t$ de student, $p<0.05$ ) (tableaux III et IV).

Au niveau de la variabilité des taux d'occupation par les adultes, trois variables ont été sélectionnées par la procédure de régression multiple progressive : la pente, la surface d'abris et le taux d'occupation de l'habitat par les adultes de truite commune. Ces trois variables expliquent $77 \%$ de la variabilité des taux d'occupation de l'habitat par les adultes de truite arc-en-ciel $(p<0.05)$. Les coefficients de corrélations partiels sont respectivement de 0.13 pour la pente, 0.46 pour les abris et 0.23 pour les taux d'occupation par les adultes de truite commune. Pour les alevins et les juvéniles, aucune variable n'a été sélectionnée.

Pour les adultes de truite commune, la procédure de régression multiple n'a sélectionné que la variable surface d'abris. Ce facteur explique $81 \%$ de la variabilité des taux d'occupation de l'habitat physique $(p<0.05)$. Pour les juvéniles et les alevins, seul le taux d'occupation par les adultes a été sélectionné, il explique respectivement $67 \%$ et $74 \%(p<0.05)$ de la variation des taux d'occupation de l'habitat par les alevins et les juvéniles.

Les facteurs susceptibles d'induire une variabilité des taux d'occupation de l'habitat physique diffèrent donc entre les deux espèces de Salmonidés étudiées. Pour les truites communes adultes, seule l'abondance des abris, fortement liée à la morphologie du lit (sous-berges), est susceptible d'induire une variation dans l'occupation de l'habitat. En revanche, pour les truites arc-en-ciel, la présence des truites communes interfère avec les caractéristiques de l'habitat physique, indiquant un effet de compétition interspécifique entre les deux espèces. 
Tableau III : Taux d'occupation par les truites arc-en-ciel (en densité (ind./ $/ \mathrm{m}^{2}$ ) et en biomasse $\left(\mathrm{g} / \mathrm{m}^{2}\right)$ ) de l'habitat physique représenté par la Surface Pondérée Utile.

Table III : Occupation by rainbow trout (density (ind. $/ \mathrm{m}^{2}$ ) and biomass $\left(\mathrm{g} / \mathrm{m}^{2}\right)$ ) of the physical habitat represented by the Weighted Usable Area.

\begin{tabular}{|c|c|c|c|c|c|c|}
\hline \multirow{3}{*}{$\begin{array}{l}\text { Stations } \\
\text { SPU en : }\end{array}$} & \multicolumn{6}{|c|}{ Truite arc-en-ciel } \\
\hline & \multicolumn{2}{|c|}{ Alevin (<100 mm) } & \multicolumn{2}{|c|}{ Juvénile (100-160mm) } & \multicolumn{2}{|c|}{ Adulte $(>160 \mathrm{~mm})$} \\
\hline & Densité & Biomasse & Densité & Biomasse & Densité & Biomasse \\
\hline 1 & 0.00 & 0.00 & 0.43 & 11.01 & 0.53 & 36.61 \\
\hline 11 & 0.00 & 0.00 & 0.14 & 3.94 & 0.16 & 11.78 \\
\hline III & 0.00 & 0.00 & 0.00 & 0.00 & 0.00 & 0.00 \\
\hline IV & 0.00 & 0.00 & 1.06 & 36.40 & 0.63 & 39.76 \\
\hline V & 0.10 & 0.54 & 0.11 & 3.57 & 0.05 & 3.25 \\
\hline VI & 0.07 & 0.33 & 0.20 & 6.51 & 0.15 & 11.35 \\
\hline VII & 0.00 & 0.00 & 0.71 & 25.28 & 0.65 & 37.29 \\
\hline VIII & 0.00 & 0.00 & 0.00 & 0.00 & 0.27 & 20.81 \\
\hline IX & 0.00 & 0.00 & 0.00 & 0.00 & 0.49 & 22.69 \\
\hline$x$ & 0.36 & 2.32 & 1.43 & 43.17 & 0.43 & 22.60 \\
\hline$X I$ & 0.93 & 0.84 & 1.18 & 47.27 & 1.04 & 62.40 \\
\hline XII & 0.73 & 5.44 & 0.51 & 17.03 & 0.61 & 40.76 \\
\hline XIII & 0.51 & 8.09 & 0.84 & 25.98 & 0.57 & 40.62 \\
\hline XIV & 0.13 & 0.89 & 1.07 & 32.95 & 0.68 & 43.95 \\
\hline$x V$ & 0.42 & 3.24 & 1.25 & 37.64 & 0.47 & 33.60 \\
\hline$X V I$ & 0.23 & 1.77 & 2.43 & 70.26 & 0.61 & 47.80 \\
\hline XVII & 0.45 & 3.37 & 0.44 & 10.96 & 0.47 & 42.94 \\
\hline
\end{tabular}

Tableau IV : Taux d'occupation par les truites communes (en densité (ind./ $\mathbf{m}^{2}$ ) et en biomasse $\left(\mathrm{g} / \mathrm{m}^{2}\right)$ ) de l'habitat physique représenté par la Surface Pondérée Utile.

Table IV : Occupation by brown trout (density (ind./ $/ \mathrm{m}^{2}$ ) and biomass $\left(\mathrm{g} / \mathrm{m}^{2}\right)$ ) of the physical habitat represented by the Weighted Usable Area.

\begin{tabular}{|c|c|c|c|c|c|c|}
\hline \multirow{2}{*}{$\begin{array}{l}\text { Stations } \\
\text { SPU en : }\end{array}$} & \multicolumn{2}{|c|}{ Alevin $(<100 \mathrm{~mm})$} & \multicolumn{2}{|c|}{$\begin{array}{c}\text { Truite commune } \\
\text { Juvénile }(100-160 \mathrm{~mm})\end{array}$} & \multicolumn{2}{|c|}{ Adulte $(>160 \mathrm{~mm})$} \\
\hline & Densité & Biomasse & Densité & Biomasse & Densité & Biomasse \\
\hline I & 0.00 & 0.00 & 0.56 & 14.34 & 0.39 & 20.84 \\
\hline ॥ & 0.04 & 0.24 & 0.12 & 3.80 & 0.14 & 8.04 \\
\hline$\|1\|$ & 0.03 & 0.29 & 0.10 & 3.14 & 0.16 & 6.74 \\
\hline IV & 0.00 & 0.00 & 0.21 & 4.31 & 0.33 & 17.60 \\
\hline V & 0.15 & 0.94 & 0.35 & 10.99 & 0.09 & 4.71 \\
\hline VI & 0.03 & 0.10 & 0.32 & 8.77 & 0.18 & 8.30 \\
\hline VII & 0.00 & 0.00 & 0.04 & 0.98 & 0.00 & 0.00 \\
\hline VIII & 0.03 & 0.15 & 0.29 & 7.45 & 0.26 & 12.43 \\
\hline $\mid X$ & 0.17 & 1.13 & 0.24 & 6.55 & 0.00 & 0.00 \\
\hline$x$ & 0.00 & 0.00 & 0.89 & 20.06 & 0.33 & 14.77 \\
\hline$X \mid$ & 0.07 & 0.80 & 0.24 & 8.28 & 0.23 & 11.47 \\
\hline
\end{tabular}




\section{DISCUSSION}

La compétition interspécifique entre Salmonidés peut jouer un rôle vis-à-vis de l'occupation de l'habitat physique. Des reductions diabondances ou de croissance, des changements de niches écologiques sont des indicateurs de compétition entre espèces (WERNER et HALL, 1976 ; DIAMOND. 1978 ; MacNALLY, 1983). Dans le ruisseau d'Estibère, les réductions d'abondances de truites arc-en-ciel en sympatrie avec les truites communes montrent qu'il existe une interaction entre les deux espèces. LOAR et al. (1985) indiquent des biomasses et densités de truite arc-en-ciel significativement inférieures dans des situations de sympatrie avec des truites communes par rapport à des situations d'allopatrie. Toutefois, les différences d'abondances peuvent être liées aux conditions d'habitat. L'analyse du taux d'occupation de l'habitat physique permet de s'affranchir de ce problème et confirme dans le cas du ruisseau d'Estibère une compétition interspécifique marquée entre les deux espèces de Salmonidés. L'effet de la compétition semble nettement plus marqué pour la truite arc-en-ciel que pour la truite commune. Cette dernière est en effet considérée comme la plus aggressive des espèces de Salmonidés se plaçant très souvent en situation de dominance par rapport aux espèces de la même famille (KALLEBERG, 1958 ; NYMAN, 1970 ; FAUSCH et WHITE, 1981 ; WATERS 1983 ; GATZ et al., 1987).

Les taux d'occupation de l'habitat pour la truite commune ne sont influencés que par l'abondance des abris. L'abondance et l'occupation de l'habitat par les truites arc-en-ciel ne jouent aucun rôle. Les truites communes sont fortement liées aux abris (LEWIS, 1969 ; BJORNN, 1971 ; HUNT, 1976 ; WESCHE et al., 1987, BARAN et al., 1993a et 1993b). Dans le cas du ruisseau d'Estibère, les abris sont essentiellement constitués par des sous-berges et donc dépendants de la morphologie du lit. La granulométrie intervient peu en terme de caches. Elle ne sera donc que très peu prise en compte par la méthode des microhabitats. Ces résultats confirment que pour un secteur déterminé de rivière, avec des conditions physico-chimiques identiques, les abondances de truite commune adulte sont très fortement influencées par les caractéristiques de l'habitat physique échantillonnées à l'étiage.

En sympatrie, la pente, les caches et l'abondance des truites communes influencent l'occupation de l'habitat par les truites arc-en-ciel. FAUSCH (1989) montre que la pente du cours d'eau peut avoir un effet significatif sur la compétition entre Salmonidés. Dans les zones de forte pente, la dominance de l'omble de fontaine (Salvelinus fontinalis) habituellement observé sur le "cou coupé" (Oncorhynchus clarkii) est très nettement inversée. On rencontre le même type d'observations entre la truite arc-en-ciel et l'omble de fontaine (FAUSCH, 1988). L'autéocologie de la truite commune et de la truite arc-en-ciel est assez bien connue (RALEIGH et al., 1984 et 1986), tandis que leur synécologie est nettement moins documentée (GATZ et al., 1987). Ces deux espèces possèdent des préférences d'habitat très proches (HARTMAN 1963 ; BUTLER et HAWTHORNE, 1968 ; JENKINS, 1969 ; LEWIS, 1969 ; SHIRVELL et DUNGEY, 1983). ELLIOT (1973) montre que dans le ruisseau d'Estibère, le régime alimentaire des deux espèces est très proche. Ce chevauchement de niche et leur évolution allopatrique (la truite arc-en-ciel dans l'ouest des Etats-Unis et la truite commune en Europe) sont autant de facteurs favorables à l'établissement d'une compétition forte entre les deux espèces. GATZ et al. (1987) indiquent une modification des microhabitats sélectionnés par la truite arc-en-ciel en présence de truite commune. Ils observent un déplacement des poissons vers des microhabitats moins favorables du point de vue énergétique (vitesse plus rapide). Dans le ruisseau d'Estibère, en sympatrie, les abondances de truite arc-en-ciel augmentent avec la valeur de vitesse moyenne, ce qui confirmerait les observations de microhabitats citées ci-dessus. HAYES (1989) indique que les alevins de truite commune (43-67 mm) dominent les alevins de truite arc-en-ciel, alors que la situation est inversée pour des alevins de taille $<15 \mathrm{~mm}$. Dans le ruisseau d'Estibère, la reproduction de la truite arc-enciel a lieu au printemps (DUMAS, 1976 ; DELACOSTE, données non publiées), ce qui limite la compétition entre les stades alevins $<15 \mathrm{~mm}$; en revanche, pour des tailles supérieures, la compétition entre les deux espèces est possible en fin de période estivale et s'exprimera en faveur de la truite commune.

La compétition interspécifique peut influencer l'interprétation des résultats de modèles d'habitat. Pour la truite commune, il semble qu'elle ait une influence réduite pour le type de rivière de montagne étudié et en présence d'une autre espèce de la même famille. En effet, HELAND (1973) ou encore GAUDIN et HELAND (1984) montrent que les 
alevins de truites communes peuvent entrer en compétition avec le vairon ou le chabot. La présence d'autres espèces (Salmonidés ou Cyprinidés rhéophiles, par exemple) doit donc être envisagée dans la définition de la capacité d'accueil d'un cours d'eau. De plus, il apparaît indispensable d'approfondir les connaissances dans le domaine des mécanismes biologiques impliqués dans la compétition (FAUSCH, 1988). Des expériences complémentaires doivent être conduites afin de mieux prévoir les effets des introductions de Salmonidés ou d'autres espèces dans des cours d'eau de la zone trutticole.

\section{REMERCIEMENTS}

Cette étude a été réalisée grâce à la participation financière du Parc National des Pyrénées Occidentales. Les auteurs tiennent tout particulièrement à remercier $\mathrm{M}$. Gérard UZABIAGA, garde du Parc National, pour ses informations et sa participation active aux travaux de terrain.

\section{BIBLIOGRAPHIE}

BARAN P., DELACOSTE M., LASCAUX J.M., BELAUD A., 1993a. Relations entre les caractéristiques de l'habitat et les populations de truite commune (Salmo trutta L.) de la vallée de la Neste d'Aure. Bull. Fr. Pêche Piscic., 331, 321-340.

BARAN P., DAUBA F., DELACOSTE M., LASCAUX J.M., 1993b. Essais d'évaluation quantitative du potentiel halieutique d'une rivière à Salmonidés à partir des données de I'habitat physique. In GASCUEL D., DURAND J.L., FONTENEAU A., Les recherches françaises en évaluation quantitative, et modélisation des ressources et systèmes halieutiques, 15-38, éditions de l'ORSTOM, Colloque et Séminaire, Paris.

BELAUD A., CHAVEROCHE P., LIM P., SABATON C., 1989. Probability-of-use curves applied to brown trout (Salmo trutta L.) in rivers of Southern France. Regulated rivers : Research and management, $\mathrm{Vol} 3,321-336$.

BJORNN T.J., 1971. Trout and salmon movements in two Idaho streams as related to temperature, food, stream flow, cover and population density. Trans. Am. Fish. Soc., 100 (3), 423-438.

BOVEE K.D., 1982. A guide to stream habitat analysis using Instream Flow Incremental Methodology. Instream Flow Information Paper $n^{\circ}$ 12, FWS/OBS 82/86, 248 p.

BOZEK M.A., HUBERT W.A., 1992. Segregation of resident trout in streams as predicted by three habitat dimensions. Can. J. Zool., vol 70, 886-890.

BUTLER R.L., HAWTHORNE V.M., 1968. The reactions of dominant trout to changes in overhead artificial cover. Trans. Am. Fish. Soc., 97, 37-41.

DIAMOND J.M., 1978. Niche shifts and rediscovery of interspecific competition. Amer. Sci., $66,322-331$.

DUMAS J., 1976. Dynamique et sédentarité d'une population naturalisée de truite arc-enciel (Salmo gairdneri Richardson) dans un ruisseau de montagne, l'Estibère (HautesPyrénées). Ann. Hydrobiol., 7 (2), 115-139.

ELLIOT J.M., 1973. The food of brown and rainbow trout (Salmo trutta and Salmo gairdneri) in relation to the abundance of drifting of invertebrate in a mountain stream. Oecologia, 12, 329-347.

FAUSCH K.D., 1988. Tests of competition between native and introduced salmonids in streams. What have we learned? Can. J. Fish. Aquat. Sci., 45, 2238-2246.

FAUSCH K.D., 1989. Do gradient and temperature affect distributions of, and interactions between, brook charr (Salvelinus fontinalis) and other resident salmonids in streams ? In KAWANABE H., YAMAZAKI F., NOAKES D.L.G., biology of charrs and masu salmon. Physiol. Ecol. Japan, Spec, vol 1, 303-322. 
FAUSCH K.D., WHITE R.J., 1981. Competition between brook trout (Salvelinus fontinalis) and brown trout (Salmo trutta) in a Michigan stream. Can. J. Fish Aquat. Sci., 38, 1220-1227.

FAUSCH K.D., WHITE R.J., 1986. Competition among juveniles of coho salmon, brook trout, and brown trout in a laboratory stream, and implications for great lakes tributaries. Trans. Am. Fish. Soc., 115, 363-381.

GATZ J., SALE A.J., LOAR J.M., 1987. Habitat shifts in rainbow trout : competitive influence of brown trout. Oecologia, 74, 7-19.

GAUDIN P., HELAND M., 1984. Influence d'adultes de chabot (Cottus gobio L.) sur des alevins de truite commune (Salmo trutta L.) : étude expérimentale en milieux seminaturels. Acta Oecol. Appl., 5 (1), 71-83.

HARTMAN G.F., 1963. Observations on behavior of juvenile brown trout in a stream aquarium during winter and spring. J. Fish. Res. Board. Can., 20, 769-787.

HAYES J.W., 1989. Social interactions between $0+$ brown and rainbow trout in experimental stream troughs. New Zeal. J. Mar. Fres. Res., 23, 24-31.

HELAND M., 1973. Observations préliminaires sur la compétition interspécifique entre le vairon Phoxinus phoxinus L. et l'alevin de truite commune Salmo trutta L.. Bull. Fr. Piscic., 250, 5-16.

HUNT R.L., 1976. A long-term evaluation of trout habitat development and its relation to improving management-related research. Trans. Am. Fish. Soc., 105 (3), 361-364.

JENKINS T.M.Jr., 1969. Social structure, position choice and microdistribution of two trout species (Salmo trutta and Salmo gairdneri) resident in mountain streams. Anim. Behav. Monog., 2, 55-123.

KALLEBERG H., 1958. Observations in a stream tank of territoriality and competition in juvenile salmon and trout (Salmo trutta L. and Salmo gairdneri L.). Rep. Inst. Freshwater Res. Drottningholm, 39, 55-88.

LEWIS S.L., 1969. Physical factors influencing fish populations in pool of a trout stream. Trans. Am. Fish. Soc., 115, 183-195.

LOAR J.M., SALE M.J., CADA G.F., 1985. Application of habitat evaluation in southern Appalachian trout stream. Environmental Sciences Division publication, 2383, ORNL/TM. 9323, $230 \mathrm{p}$.

MCNALLY R.C., 1983. On assessing the significance of interspecific competition to guild structure. Ecology, 64, 1646-1652.

NYMAN O.L., 1970. Ecological interaction of brown trout, Salmo trutta L., and brook trout, Salvelinus fontinalis (Mitchill), in a stream. Can. Field-Natur., 84, 343-350.

RALEIGH R.F., ZUCKERMAN L.D., SOLOMON R.C., NELSON P.C., 1984. Habitat suitability information : Rainbow trout. U.S. Fish. Wild. Ser. Biol. Rep. FWS/OBS-82 (10-60), 64 p.

RALEIGH R.F., ZUCKERMAN L.D., NELSON P.C., 1986. Habitat suitability index models and stream flow suitability curves : brown trout, revised. U.S. Fish. Wild. Ser. Biol. Rep., FWS/OBS-82 (10-124), 65 p.

SHIRVELL C.S., DUNGEY R.G., 1983. Microhabitats chosen by brown trout for feeding and spawning in rivers. Trans. Am. Fish. Soc., 112, 355-367.

WATERS T.F., 1983. Replacement of brook trout by brown trout over 15 years in a Minnesota stream : production and abundance. Trans. Am. Fish. Soc., 112, 137-146.

WEISBERG S., 1980. Applied linear regression. John Wiley and Sons, New York, 283 p.

WERNER E.E., HALL D.J., 1976. Niche shifts in sunfish : experimental evidence and significance. Science, 191, 404-406.

WESCHE T.A., GOERTLER C.M., FRYE C.B., 1987. Contribution of riparian vegetation to trout cover in small streams. N. Am. J. Fish. Manage., 7, 151-153. 